\title{
Hypergraphia in epilepsy: is there a specificity to temporal lobe epilepsy?
}

\author{
BRUCE P HERMANN, ${ }^{*}$ STEVEN WHITMAN, $†$ PAUL ARNTSON $\dagger$
}

From the Depurtment of Neurology, University of Illinois College of Medicine at Chicago, the Center for Urban Affairs and Policy Research, and Department of Communications, Northwestern University, Chicago, Illinois, USA

SUMMARY The purpose of this investigation was to determine the specificity of hypergraphia to temporal lobe epilepsy using the paradigm developed by Sachdev and Waxman (1981). One hundred and thirty-eight patients were sent a standard stimulus letter and the response rates were examined for the patient groups with temporal lobe epilepsy $(\mathrm{N}=80)$, and without $(\mathrm{N}=31)$, and mixed seizure types $(\mathrm{N}=13)$. For those patients who responded, additional analyses involved the number of words per response and the presence/absence of any qualitative indices of the postulated interictal behavioural syndrome of temporal lobe epilepsy. The results were interpreted in the light of the contemporary definitions of hypergraphia and were related to the larger literature concerned with personality and behavioural change in temporal lobe epilepsy.

Investigations of the relationship between temporal lobe epilepsy and disorders of interictal behaviour generally fall into one of six areas which can be characterised by the personality and/or behaviour problem under study: (1) psychosis; (2) aggression; (3) sexual dysfunction; (4) depression and anxiety; (5) general psychopathology as assessed by standardised personality tests or other measures of problem behaviour (for example, rates of psychiatric hospitalisation); and (6) non-psychopathological behaviour and personality change. Issues in this last category have recently attracted much attention.

Although the concept of a global "epileptic personality" has generally fallen into disfavour for lack of support from well-controlled investigations, several case studies and anecdotal observations have raised interest in unusual personality and behavioural characteristics posited to bear a relationship with temporal lobe epilepsy (for example, sudden religious conversions, increased philosophi-

\footnotetext{
Address for reprint requests: Dr Hermann, Department of Neurology, University of Illinois at Chicago, 912 South Wood Street, 855 North, Chicago, IL 60612, USA.

*Supported in part by Grant MCJ 173029-01-0 from the Department of Health and Human Services, MCH/FP, Region V.

tSupported by the Epilepsy in the Urban Environment Project, Center for Urban Affairs and Policy Research, Northwestern University.
}

Received 2 March 1983.

Accepted 16 April 1983. cal interest, humourlessness). Recently, Waxman and Geschwind proposed the existence of a specific interictal behavioural syndrome associated with temporal lobe epilepsy consisting primarily of alterations in sexual behaviour, increased religiosity, and a tendency toward extensive and oftentimes compulsive writing (hypergraphia). ${ }^{12}$ Bear elaborated upon this syndrome and proposed the existence of 18 traits associated with temporal lobe epilepsy which reflected alterations in behaviour, thought, and affect. ${ }^{3}$ It was suggested that these traits were related to an underlying mechanism-enhanced affective associations to previously neutral stimuli, events or concepts-thought to be caused by a progressive change in limbic system structure secondary to a temporal epileptic focus. Bear specifically argued that an epileptiform focus in the limbic system produced new functional connections between neocortical and limbic structures and he called this process sensory-limbic hyperconnection.

Geschwind $^{4}$ and Bear $^{3}$ argued that as these behavioural changes were neither maladaptive nor psychopathologic in the traditional sense, new and/ or different assessment techniques were needed to demonstrate their existence. Eight studies of behavioural change in temporal lobe epilepsy and of the proposed underlying mechanisms have progressed along four investigative lines which can be grouped according to the dependent measures used: (a) specially designed personality questionnaires, that is, the Personal Inventory and Personal 
Behaviour Survey; $;^{5-9}$ (b) measures of physiological responsivity to stimuli varying in the nature and degree of affective content; ${ }^{10}$ (c) newly developed semi-structured psychiatric interview protocols; ${ }^{11}$ and (d) direct measures of behaviour. ${ }^{12}$

This last category is particularly interesting as it represents an attempt to confirm objectively a specific aspect (hypergraphia) of the postulated interictal syndrome of temporal lobe epilepsy. Further, it is methodologically unique because, instead of asking the patient or others to determine or verify the presence/absence of hypergraphia via a variety of self-report or rater techniques, it relies on relatively unobtrusive measures of the patient's actual writing behaviour.

Sachdev and Waxman ${ }^{12}$ wrote to 63 previous in-patients with epilepsy or seizure disorder as the primary or secondary discharge diagnosis and asked them to write and "... describe to the best of your ability, your present state of health, understanding of the disease (seizure disorder), and the changes in your life resulting from it" (p 358). Seventeen responses were received and the letters were analysed as to: (1) the number of words per letter, and (2) the degree of manifestation of the postulated interictal syndrome reflected in the content of the letter (probable, possible, no evidence). Comparisons were made between the responders with $(\mathrm{N}=9)$ and without $(\mathrm{N}=8)$ temporal lobe epilepsy. Seizure type variations in responder/nonresponder status were also investigated. Proportionately more of the temporal lobe epilepsy patients were responders, manifested more of the interictal syndrome via the content of the letters, and wrote significantly longer letters.

The specificity of hypergraphia to temporal lobe epilepsy is, however, still open to question as the non-temporal lobe epilepsy group of Sachdev and Waxman was composed largely of individuals without other forms of epilepsy. The majority of the non-temporal lobe epilepsy group (30 of 34) had a normal EEG or an abnormal but non-epileptiform EEG. Further, many of the non-temporal lobe epilepsy patients (22 of 34 ) suffered from withdrawal seizures and five patients had seizures in association with a metabolic encephalopathy. Only four of 34 patients in the non-temporal lobe epilepsy group had primary generalised epilepsy documented by a corticoreticular EEG pattern. Further, the influence of potentially relevant confounding variables was not considered.

The purpose of this investigation was to replicate the study of Sachdev and Waxman, correcting for the methodological issues noted above, in order to assess the specificity of hypergraphia to temporal lobe epilepsy.

\section{Method}

Subjects for this study consisted of 157 patients attending the Consultation Clinic for Epilepsy of the Department of Neurology, University of Illinois at Chicago, for treatment and/or evaluation of known or suspected epilepsy. The Director of the clinic and the EEG Laboratory independently classified the patients' seizure type according to the International Classification System and coded the results of the waking and sleeping EEG investigations, medication type and dosage, as well as the average seizure frequency for each patient. Additional demographic (age, sex, race) and seizure-related (duration of disorder) data were retreived by the present investigators. These 157 patients represented virtually a consecutive series of patients; six were omitted because they were Spanish speaking only and a small undetermined number were omitted because their records were unavailable.

For the purposes of this investigation, the records of these 157 patients were reviewed prior to our contacting them by mail. Nineteen patients were excluded from further consideration; nine had non-epileptiform EEGs (small sharp spikes, positive spikes, focal slow waves only), four had no EEG performed; five had inadequate information in their records and one had narcolepsy only. A final sample pool of 138 patients remained. Ninety of the patients had temporal lobe epilepsy (complex partial and/ or secondarily generalised seizures with a spike or sharp wave focus in one or both temporal lobes), 29 had generalised epilepsy (for example, primary generalised tonicclonic and/or absence seizures with a corticoreticular EEG pattern (four patients in this group had a 6/s spike and wave pattern while the remaining had the more common $3 /$ cps spike and wave pattern), four had focal non-temporal seizures (simple partial and/or secondarily generalised seizures with a focal but non-temporal lobe spike focus), and 15 had mixed seizure types, all but one of which had a combination of temporal lobe epilepsy and generalised epilepsy. For the purposes of this investigation the patients with generalised and focal non-temporal lobe epilepsy were combined and denoted non-temporal lobe epilepsy. These 138 people were then sent the letter developed by Sachdev and Waxman which was provided to us by Waxman.

The response rate and mean number of words per letter were computed for the temporal lobe epilepsy, nontemporal lobe epilepsy and mixed seizure groups. In addition, the responses were checked for the presence or absence of reference to meaning or significance of seizures, philosophical, ethical or religious themes, and unusual style (calligraphy, use of unusual symbols or drawings) (ref $12, p 358$ ) by an independent rater blind to the purposes of this study as well as to the demographic and seizure-related characteristics of each responder.

As noted, four of the 29 patients with seizures in the non-temporal lobe epilepsy group demonstrated a $6 / 5$ spike and wave pattern. Some investigators consider this bilaterally synchronous and symmetrical waveform to be consistent with a corticoreticular type of pattern closely associated with epilepsy. ${ }^{13}$ Others remain uncertain of this waveform's diagnostic significance. ${ }^{14} \mathrm{We}$ will thus present the data for the non-temporal lobe epilepsy patients both 
Table 1 Characteristics of seizure type subgroups

\begin{tabular}{|c|c|c|c|}
\hline \multirow[t]{2}{*}{ Characteristic } & \multicolumn{3}{|l|}{ Group } \\
\hline & Temporal lobe epilepsy $(N=80)$ & Non-temporal lobe epilepsy $(N=31)$ & Mixed $(N=13)$ \\
\hline $\begin{array}{l}\text { Sex } \\
\text { \%Female } \\
\text { \%Male }\end{array}$ & $\begin{array}{l}45 \\
55\end{array}$ & $\begin{array}{l}48 \\
52\end{array}$ & $\begin{array}{l}69 \\
31\end{array}$ \\
\hline $\begin{array}{l}\text { Race } \\
\text { \%Black } \\
\text { \%White }\end{array}$ & $\begin{array}{l}26 \\
74\end{array}$ & $\begin{array}{l}29 \\
71\end{array}$ & $\begin{array}{l}15 \\
85\end{array}$ \\
\hline $\begin{array}{l}\text { Seizure Control } \\
\text { \%Good } \\
\text { \%Fair } \\
\text { \%Poor }\end{array}$ & $\begin{array}{l}25 \\
52 \\
23\end{array}$ & $\begin{array}{l}56 \\
27 \\
17\end{array}$ & $\begin{array}{l}31 \\
46 \\
23\end{array}$ \\
\hline $\begin{array}{l}\text { Age } \\
\text { Average }\end{array}$ & 42 & 33 & 36 \\
\hline $\begin{array}{l}\text { Duration of Seizures } \\
\text { Average }\end{array}$ & 27 & 23 & 27 \\
\hline
\end{tabular}

with and without the inclusion of these four subjects and will refer to these groups as non-temporal lobe epilepsy and reduced non-temporal lobe epilepsy respectively.

Table 1 presents the distributions of the auxiliary variables that are considered in this paper for the three main seizure type subgroups (temporal lobe epilepsy, nontemporal lobe epilepsy, mixed). The three groups did not differ significantly according to race, sex, or duration of seizures $(p>0.05)$. However, there were some differences in the remaining variables. The seizure type control distributions differed among the three groups $(p<0.05)$ although there were no significant differences among the proportions with poor control $(p>0.05)$. The temporal lobe epilepsy group was significantly older than the nontemporal lobe epilepsy group $(p<0.001)$ but did not differ significantly from the mixed group on this variable $(p>0.05)$. Finally, there were no significant differences among the groups according to number and type of anticonvulsants currently being taken $(p>0.05)$.

\section{Results}

Table 2 summarises the main results of the study. Six of the 138 people had died and eight others had moved, leaving no forwarding address. The response rate was $24 \%(19 / 80)$ for the temporal lobe epilepsy group, $10 \%(3 / 31)$ for the non-temporal lobe epilepsy group, $(4 \%(1 / 27)$ for the reduced nontemporal lobe epilepsy group; $50 \%(2 / 4)$ for the $6 \mathrm{~s} / \mathrm{sw}$ patients in the non-temporal lobe epilepsy group) and $38 \%(5 / 13)$ for the mixed group. The differences in response rates between the temporal lobe epilepsy group and the non-temporal lobe epilepsy group and the temporal lobe epilepsy group and mixed group were not significant $(p>0.05)$, but the temporal lobe epilepsy/reduced nontemporal lobe epilepsy groups difference was significant $(p<0.05)$. The mixed group had a response rate that was significantly higher than the reduced non-temporal lobe epilepsy group $(p<0.01)$ and the non-temporal lobe epilepsy group $(p<0.05)$.

The average length of response was 296 words for the temporal lobe epilepsy group, 371 words for the non-temporal epilepsy group, (64 words for the one response in the reduced non-temporal lobe epilepsy group; 524 words for the 6/s spike and wave patients in the non-temporal lobe epilepsy group) and 260 words for the mixed group. The number of responses was too small to allow statistical comparison of the differences in mean length of letters among these groups. However, an understanding of the relationship among these distributions can be obtained from table 3 , which presents the frequency distributions of letter length for the seizure subgroups. The two letters in the temporal lobe epilepsy group that were more than $\mathbf{5 0 0}$ words in length were 1176 and 1229 words.

Table 2 Response rates and average length of letter for seizure type subgroups

\begin{tabular}{lccc}
\hline & $\begin{array}{l}\text { Temporal } \\
\text { lobe epilepsy }\end{array}$ & $\begin{array}{l}\text { Non-temporal } \\
\text { lobe epilepsy }\end{array}$ & Mixed \\
\hline $\begin{array}{l}\text { Letters mailed to } \\
\text { participants }\end{array}$ & 90 & 33 & 15 \\
$\begin{array}{l}\text { Died } \\
\text { No forwarding address }\end{array}$ & 6 & 1 & 1 \\
$\begin{array}{l}\text { Responses } \\
\text { Non-responses }\end{array}$ & 19 & 1 & 1 \\
$\begin{array}{l}\text { Response rate } \\
\text { Average length of }\end{array}$ & 61 & 3 & 5 \\
$\quad$ response & $24 \%$ & $10 \%$ & $38 \%$ \\
\hline
\end{tabular}

*Includes four patients with $6 / \mathrm{s}$ spike wave pattern, four patients with focal epilepsy, and 23 patients with generalised epilepsy. Two of the responses were from patients with the 6/s spike wave pattern ( 589 and 459 words) and one was from a patient with generalised epilepsy (64 words). The reduced non-temporal lobe epilepsy group (minus the $6 / \mathrm{s}$ spike wave patients) generated one letter (4\% response rate) which was 64 words in length. 
Table 3 Frequency distributions for length of letter for seizure type subgroups

\begin{tabular}{lclc}
\hline $\begin{array}{l}\text { Number of } \\
\text { Words }\end{array}$ & $\begin{array}{l}\text { Temporal } \\
\text { lobe epilepsy }\end{array}$ & $\begin{array}{l}\text { Non-temporal } \\
\text { lobe epilepsy }\end{array}$ & Mixed \\
\hline $0-99$ & 5 & 1 & 1 \\
$100-199$ & 7 & 0 & 1 \\
$200-299$ & 0 & 0 & 1 \\
$300-399$ & 3 & 0 & 1 \\
$400-499$ & 2 & 1 & 1 \\
$500-599$ & 0 & 1 & 0 \\
$\geqslant 600$ & $2 \dagger$ & 0 & 0 \\
Total & 19 & 3 & 5 \\
\hline
\end{tabular}

*See table 2 footnote.

†These two letters were 1176 words and 1229 words.

Finally, no letter contained any of the special qualitative features (reference to the meaning of significance of seizures, philosophical, ethical or religious themes or unusual style) hypothesised to indicate hypergraphia in temporal lobe epilepsy. ${ }^{12}$

\section{Discussion}

We begin with a discussion of some of the methodological complexities in this area of research. Within this context, we then examine differences among groups within our study. Finally, we compare our findings to those of Sachdev and Waxman. ${ }^{12}$

The initial studies hypothesising a temporal lobe epilepsy/hypergraphia specificity were interesting anecdotal case reports. ${ }^{12}$ In these reports hypergraphia was attributed to writing styles that were unusual (for example, use of mirror images, codes, different coloured inks, ritualised script, calligraphy), excessive (for example, in length of writings and/or in frequency and duration of writing behaviour), or contained references to selected themes (for example, philosophical, ethical, moral, religious). In few of these reports were any of these three characteristics (unusual style, excessiveness, selected themes) operationally defined. For example, none of the authors suggested a guide as to what length or frequency would be "excessive" or to what extent or what kind of reference to religion would be necessary to thus categorise a writing sample.

Bear and Fedio ${ }^{5}$ measured hypergraphia by asking five questions abut their subjects' writing style. This had the advantage of allowing replication but it (a) was still a subjective report from the patient and/or a significant other and (b) assessed only one characteristic of hypergraphia: excessive or frequent writing. The study by Sachdev and Waxman ${ }^{12}$ is the first study in this area that is simultaneously objective, allows for replication and considers all aspects of hypergraphia, and for that reason merits special attention.

A final conceptual and methodological issue has just recently been raised by Roberts and colleagues. ${ }^{15}$ They suggest that hypergraphia, which they maintain is uncommon, may not be a graded phenomenon which could thus be seen as characterising a distribution, but might rather be an "allor-none" phenomenon that would appear in only a few people (ref 15, p 136). If this speculation is correct, then a central aspect of studies such as this one, and the one by Sachdev and Waxman, would be inappropriate since both try to characterise distributions (by average letter length and response rate) an endeavour that would be rendered meaningless if the all-or-none perspective is appropriate.

What can be said of our results in the context of these conceptual and methodological observations? First, temporal lobe epilepsy patients demonstrated a higher response rate $(24 \%)$ than the non-temporal lobe epilepsy $(10 \%)$ and reduced non-temporal lobe epilepsy (4\%) groups but a lower response rate relative to the Mixed group (38\%). If response rate is an indication of hypergraphia (Sachdev and Waxman ${ }^{12}$ are the first ones to suggest this), then these results support the hypothesised relationship between temporal lobe epilepsy and hypergraphia as patients with a temporal lobe spike focus, either in isolation (temporal lobe epilepsy) or in combination with corticoreticular discharge (mixed), had a higher response rate relative to non-temporal lobe epilepsy patients.

The results of letter length do not support the notion of a temporal lobe epilepsy/hypergraphia specificity if hypergraphia is considered to be a graded or distributional phenomenon. The nontemporal lobe epilepsy group wrote letters that were 371 words long on the average, compared to 296 words for the temporal lobe epilepsy group and 260 words for the mixed group. The mean non-temporal lobe epilepsy letter length is mostly a function of the inclusion of four patients with $6 \mathrm{~Hz}$ spike and wave discharges, two of whom responded and wrote long letters (589 and 459 words). (See refs 13,16 for a review and discussion of the psychological concommitants of the 6/s spike and wave pattern). If these two patients are removed from the non-temporal lobe epilepsy group, then there is only one response left in that group, and it is a very short letter (64 words). However, if the patients with seizures with 6/s spike and wave discharges do not belong in the non-temporal lobe epilepsy group, they nonetheless belong somewhere, for example, in an unclassifiable group; and this group (of two) wrote letters that averaged 524 words, much longer than either the temporal lobe epilepsy or mixed group.

If, on the other hand, hypergraphia is considered to be an all-or-none behaviour, then letter length would suggest support for temporal lobe epilepsy/ 
hypergraphia specificity as the two longest letters (1176 and 1229 words) were both written by people with temporal lobe epilepsy.

Finally, the independent blind rater failed to detect any unusual themes or style in any of the letters. (The closest we could find was one letter in which circles were used over the "i's" in the place of dots). Such results neither support nor deny a temporal lobe epilepsy/hypergraphia specificity. (While excessive and extensive writing has been a central characteristic of all cases and studies of hypergraphia to date, ${ }^{1251215}$ close review of published reports indicates that a lack of unusual form and/or content in the writings does not preclude hypergraphia). However, there were 93 people with temporal lobe epilepsy in our study ( 80 had temporal lobe seizures only, and 13 had temporal lobe seizures in conjunction with other seizure types) and the fact that none produced any of these characteristics suggests that they are indeed uncommon.

The effects of auxillary variables on these comparisons among seizure type groups must be considered. As noted above, the groups were similar according to sex, race, duration of seizures and number and type of anticonvulsant medication(s) but did vary somewhat according to age and seizure control. The small sample sizes made it impossible to control statistically for these differences but it is unlikely that any one of these variables accounted for the observed differences in response rate and average length of letter. The possible effects of combinations, or interactions, of variables could not be evaluated. However, it is interesting to note that the two long temporal lobe epilepsy letters were both written by white women with good seizure control. There are, of course, other variables that should be considered but which unfortunately were unavailable to us. These would include dose and length of time on medications, education, intelligence, general neuropsychological status, rapport with clinic physicians, and so on.

There are three intriguing differences between our findings and those of Sachdev and Waxman ${ }^{12}$ : (1) they received responses from $56 \%$ of their temporal lobe epilepsy group while we received responses from only $24 \%$ of our temporal lobe epilepsy group; (2) their temporal lobe epilepsy group wrote letters that averaged 1301 words in length. Two of these letters were 5540 and 4200 words in length. Our temporal lobe epilepsy group averaged 296 words and our two longest temporal lobe epilepsy letters (1176 and 1229 words) were shorter than the average length of the Sachdev and Waxman temporal lobe epilepsy group responses; (3) they suggested the existence of a probable or possible interictal syndrome in eight of their nine temporal lobe epilepsy responses $(89 \%)$ while we detected a possible interictal syndrome in only two of our patients with temporal lobe epilepsy (2\%).

What might account for these differences? First, it has been suggested that patients with temporal lobe epilepsy with bitemporal lobe EEG foci produce more psychopathology in the form of abnormal MMPI profiles than temporal lobe epilepsy patients with unilateral temporal lobe foci. ${ }^{17}$ In the Sachdev and Waxman study, $37 \%$ of the temporal lobe epilepsy sample had bitemporal EEG foci and those patients manifested a higher response rate, a greater proportion with probable interictal syndrome, and they wrote longer letters ( 2515 words $v s 348$ words) than the temporal lobe epilepsy patients with a unilateral epileptiform focus. Our temporal lobe epilepsy sample had a much smaller proportion of patients with bitemporal lobe EEG abnormalities (5\%) and this may account in part for the differences between our temporal lobe epilepsy sample and that of Sachdev and Waxman. Our small number of bitemporal patients precludes any meaningful analyses. However, it should be noted that we received two letters from patients with bitemporal lobe foci; one was the longest (1229 words) and the other was one of the shortest (69 words). In addition, Sachdev and Waxman's temporal lobe epilepsy patients with a unilateral focus wrote longer letters than our comparable temporal lobe epilepsy patients.

A second possible explanation for these differences involves sample selection. As our series was selected from a chronic out-patient neurological setting, it is possible that "truly" hypergraphic patients may have been selected out of our sample pool and, for instance, referred to the psychiatry service for treatment of concommitant behavioural problems. Additionally, the inpatient status of the Sachdev and Waxman sample might suggest that their patients had a more severe seizure disorder relative to our out-patient sample. Perhaps hypergraphia bears some association to the severity of the seizure disorder and/or the underlying neurological condition. Finally, the unknown effects of the auxilliary variables considered above may also account for some of the observed differences between these studies as may variables we were not able to consider (for example, physician/patient rapport, medications, intelligence, education).

Do our findings support the hypothesis of a temporal lobe epilepsy/hypergraphia specificity? We think that the answer depends upon which of the four measures of hypergraphia one considers, and whether or not one views hypergraphia as a graded or all-or-none phenomenon. If hypergraphia is a graded phenomenon, then, in this study: (a) the 
measure of response rate supports the hypothesis; (b) the measure of letter length opposes the hypothesis; (c) the measure of peculiar content neither supports nor opposes the hypothesis; (d) the measure of peculiar style neither supports nor opposes the hypothesis.

If hypergraphia is an all-or-none phenomenon, then: (a) the measure of response rate has no meaning in this context; (b) the measure of letter length supports the hypothesis; (c) the measure of peculiar content neither supports nor opposes the hypothesis; (d) the measure of peculiar style neither supports nor opposes the hypothesis.

Further research in this area should attempt to determine whether hypergraphia is best conceptualised as a graded or an all-or-none phenomenon, as well as attempt to arrive at operational definitions of the main characteristics thought to reflect hypergraphia. In addition, consideration should be paid to the patient populations that are sampled. The differences between our results and those of Sachdev and Waxman ${ }^{12}$ suggest that the effect of sample selection may be substantial. Finally, we urge the examination of potentially intervening variables like those utilised in this study and others that we were not able to incorporate-like intelligence, patientphysician rapport, and dose and length of time on medications.

We wish to express our appreciation to John $\mathbf{R}$ Hughes, MD, Director of the EEG Laboratory and the Consultation Clinic for Epilepsy, for seizure type and frequency classification of the subjects in this study. We also wish to thank Jade Dell for evaluating the special features of the letters.

\section{References}

${ }^{1}$ Waxman SG, Geschwind N. The interictal behaviour syndrome of temporal lobe epilepsy. Arch Gen Psychiatry 1975;32:1580-6.

${ }^{2}$ Waxman SG, Geschwind N. Hypergraphia in temporal lobe epilepsy. Neurology (Minneap) 1974;24:629-36.

${ }^{3}$ Bear D. Temporal lobe epilepsy: A syndrome of sensory-limbic hyperconnection. Cortex 1979;15: 357-84.

${ }^{4}$ Geschwind N. Behavioural change in temporal lobe epilepsy. Arch Neurol 1977;34:453.

${ }^{5}$ Bear D, Fedio P. Quantitative analysis of interictal behaviour in temporal lobe epilepsy. Arch Neurol 1977;34:454-67.

${ }^{6}$ Hermann BP, Riel P. Interictal personality and behavioural traits in temporal lobe and generalised epilepsy. Cortex 1981;17:125-8.

${ }^{7}$ Mungas D. Interictal behaviour abnormality in temporal lobe epilepsy: a specific syndrome or non-specific psychopathology? Arch Gen Psychiatry 1982;39: 108-11.

${ }^{8}$ Nielsen $H$, Kristensen $O$. Personality correlates of sphenoidal EEG-foci in temporal lobe epilepsy. Acta Neurol Scand 1981;64:289-300.

${ }^{9}$ Rodin E, Schmaltz S. The Bear-Fedio personality inventory and temporal lobe epilepsy. Epilepsia 1983;24:260.

${ }^{10}$ Bear D, Schenk L, Benson H. Autonomic responses to neutral and emotional stimuli in patients with temporal lobe epilepsy. Am J Psychiatry 1981;138:843-5.

${ }^{11}$ Bear D, Levin K, Blumer D, Chetham D, Ryder J. Interictal behaviour in hospitalised temporal lobe epileptics: relationship to idiopathic psychiatric syndromes. J Neurol Neurosurg Psychiatry 1982;45:481-8.

${ }^{12}$ Sachdev HS, Waxman SG. Frequency of hypergraphia in temporal lobe epilepsy: an index of interictal behaviour syndrome. J Neurol Neurosurg Psychiatry 1981;44:358-60.

${ }^{13}$ Hughes JR. Two forms of the 6/Sec spike and wave complex. Electroencephalography Clin Neurophys 1980;48:535-50.

${ }^{14}$ Maulsby RL. Patterns of uncertain significance. In: Klass DW, Daly DD, eds. Current Practice of Clinical Electroencephalography 1979. New York: Raven Press, 411-9.

15 Roberts JKA, Robertson MM, Trimble MR. The lateralizing significance of hypergraphia in temporal lobe epilepsy. J Neurol Neurosurg Psychiatry 1982;45:131-8.

${ }^{16}$ Olson SF, Arbit J, Hughes JR. Psychological testing in patients with $6 \mathrm{~s} / \mathrm{sec}$ spike and wave pattern: A controlled study. Clinical EEG 1971;2:202-5.

${ }^{17}$ Meier MJ, French LA. Some personality correlates of unilateral and bilateral EEG abnormalities in psychomotor epileptics. J Clin Psychol 1965;21:3-9. 